PROCEEDINGS OF THE

AMERICAN MATHEMATICAL SOCIETY

Volume 137, Number 11, November 2009, Pages 3619-3625

S 0002-9939(09)09946-8

Article electronically published on May 29, 2009

\title{
GEOMETRIC PROPERTIES DERIVED FROM GENERIC INITIAL SPACES
}

\author{
GUNNAR FLØYSTAD AND MIKE STILLMAN
}

(Communicated by Bernd Ulrich)

\begin{abstract}
For a vector space $V$ of homogeneous forms of the same degree in a polynomial ring, we investigate what can be said about the generic initial ideal of the ideal generated by $V$, from the form of the generic initial space $\operatorname{gin} V$ for the revlex order. Our main result is a considerable generalisation of a previous result by the first author.
\end{abstract}

\section{INTRODUCTION}

The generic initial ideal for the revlex order is a basic invariant of a homogeneous ideal $I$ in a polynomial ring. The computational complexity of the ideal is reflected in this generic initial ideal, as well as algebraic and geometric properties of the original ideal. For instance, this generic initial ideal has the same regularity as the original ideal [1]. Also the original ideal is Cohen-Macaulay iff this generic initial ideal is Cohen-Macaulay. In [5], M. Green shows that if $\operatorname{gin}(I)$ has no generator in degree $e$ and $I_{<e}$ is the subideal of $I$ generated by elements of degree less than $e$, then the generic initial ideal of this subideal is in fact equal to $\operatorname{gin}(I)_{<e}$, forcing strong conditions on the geometry of the scheme defined by $I$. With such basic properties of the ideal reflected in the generic initial ideal it is of considerable interest to understand more of what properties of the original ideal are reflected in the generic initial ideal for the revlex order.

We consider subspaces $V$ of homogeneous forms in a polynomial ring $\mathbb{k}\left[x_{1}, \ldots, x_{n}\right]$ and investigate what geometric consequences one may derive about the vanishing locus of $V$ from its generic initial space gin $(V)$, for the revlex order. In particular our results will apply to the ideal generated by the forms in $V$.

A result by the first author [3] says that if $V$ is a linear subspace of forms of degree $a+b$ and its generic initial subspace for the revlex order has as basis of monomials, the strongly stable set of monomials of degree $a+b$ generated by $x_{1}^{a} x_{m}^{b}$, where $m \geq 3$, then the polynomials of $V$ have a common factor of degree $a$.

We give a generalisation of this, Theorem 2.4. putting a considerably weaker condition on $\operatorname{gin}(V)$ and from this deriving conclusions about the growth of the Hilbert function of the ideal generated by $V$. For instance, the case when $m=n$ above is generalised as follows. The property we impose is that every monomial of

Received by the editors May 1, 2008, and, in revised form, February 25, 2009.

2000 Mathematics Subject Classification. Primary 13P10.

Key words and phrases. Generic initial ideal, revlex order.

The second author was partially supported by NSF Grant DMS-0311806.

(C)2009 American Mathematical Society Reverts to public domain 28 years from publication 
$\operatorname{gin}(V)$ is a multiple of a monomial in $\operatorname{gin}(V): x_{n}$. This enables us to conclude that the image in $\mathbb{k}\left[x_{1}, \ldots, x_{n-1}\right]$ of the ideal generated by $V$ and the ideal generated by $\operatorname{gin}(V)$ have the same Hilbert function.

In the end we consider the example where $\operatorname{gin}(V)$ has as basis the monomials $x_{1}^{3}, x_{1}^{2} x_{2}, x_{1} x_{2}^{2}, x_{1}^{2} x_{3}$ of $\mathbb{k}\left[x_{1}, x_{2}, x_{3}\right]$. We give examples showing that in this case the vanishing locus of $V$ may consist of six points, three points, or be empty.

\section{The RESUlts}

We first recall basic notions and properties of the revlex order and initial ideals. Then we formulate the main results, and in the end we give some examples and open ends.

2.1. The revlex order. We consider the revlex order on monomials in the polynomial ring $\mathbb{k}\left[x_{1}, \ldots, x_{n}\right]$ over a field $\mathbb{k}$ of characteristic zero. That is,

$$
x_{1}^{i_{1}} \cdots x_{n}^{i_{n}}>x_{1}^{j_{1}} \cdots x_{n}^{j_{n}}
$$

if either the total degree of the former is less than the total degree of the latter or, if their total degrees are equal, for the largest index $r$ such that $i_{r}$ and $j_{r}$ differ, we have $i_{r}<j_{r}$.

For an ideal $I$ in the polynomial ring, we get the initial ideal in $(I)$ generated by the largest monomials in each polynomial of $I$. Also for any vector subspace $V$ of the polynomial ring, we get a subspace in $(V)$, generated as a vector space by the largest monomials in the polynomials of $V$.

For a vector subspace $V$ of $\mathbb{k}\left[x_{1}, \ldots, x_{n}\right]$, the image of $V$ by the natural map

$$
\mathbb{k}\left[x_{1}, \ldots, x_{n}\right] \rightarrow \mathbb{k}\left[x_{1}, \ldots, x_{n-r}\right]
$$

is denoted by $V_{\mid x_{n}, \ldots, x_{n-r+1}}$ and called the restriction of $V$ to $\mathbb{k}\left[x_{1}, \ldots, x_{n-r}\right]$. If $I$ is an ideal, its restriction is again an ideal.

Two standard facts about the revlex order are the following:

i. $\operatorname{in}\left(V_{\mid x_{n}}\right)=\operatorname{in}(V)_{\mid x_{n}}$,

ii. $\operatorname{in}\left(V: x_{n}\right)=\operatorname{in}(V): x_{n}$.

2.2. Generic initial spaces. If a sequence of polynomials are surrounded by brackets \langle\rangle it will denote the vector space generated by these polynomials. If $g$ in $G L\left(\left\langle x_{1}, \ldots, x_{n}\right\rangle\right)$ is a change of coordinates, there is an open subset of such $g$ 's such that in $(g . V)$ is constant. This initial space is called the generic initial space of $V$ and denoted $\operatorname{gin}(V)$. It is a standard fact (see 2] or, originally, 4]) that $\operatorname{gin}(V)$ is strongly stable; i.e. if $m$ is a monomial in $\operatorname{gin}(V), x_{j}$ divides $m$ and $i<j$, then $x_{i} m / x_{j}$ is also in $\operatorname{gin}(V)$.

Let us recall the following result by the first author [3].

Theorem 2.1. Let $V \subseteq S_{b+a}$ be a linear subspace such that

$$
\operatorname{gin}(V)=\left\langle x_{1}, \ldots, x_{m}\right\rangle^{b} \cdot x_{1}^{a} \subseteq S_{b+a},
$$

where $m \geq 3$. Then there exists a polynomial $p$ in $S$ of degree a such that $p$ is a common factor of the elements of $V$.

The theorem we prove here is a considerable generalisation of this. First let us give an illustrative example of this generalisation.

Example 2.2. Suppose $V$ is a subspace of $\mathbb{k}\left[x_{1}, x_{2}, x_{3}\right]_{a+b+1}$ such that $x_{1}^{a} x_{2}^{b+1}$ is not in $\operatorname{gin}(V)$, but $x_{1}^{a+1} x_{2}^{b-1} x_{3}$ is in $\operatorname{gin}(V)$ (and so also $x_{1}^{a+1} x_{2}^{b}$ ). This means that 
$\operatorname{gin}(V)$ has the form given in the following figure, where an $\mathrm{x}$ denotes that the monomial is in $\operatorname{gin}(V)$ and a circle that it is not:

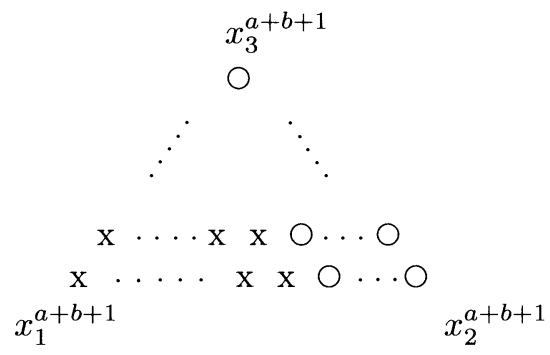

By the result of the first author, Theorem 2.1, if $\operatorname{gin}(V)$ is $x_{1}^{a+1} \cdot\left\langle x_{1}, x_{2}, x_{3}\right\rangle^{b}$, then $V$ has a common factor $p$ of degree $a+1$. But this assumption on $V$ is very strong. The assumption above is much weaker, and we shall show that it still allows us to conclude that $V$ has a common factor.

In addition to the theorem above, the following [5, Proposition 28], due to M. Green, is a main inspiration for what we prove.

Theorem 2.3. Let I be an ideal in $\mathbb{k}\left[x_{1}, \ldots, x_{n}\right]$ generated in degrees $\leq d$. Suppose in $(I)$ is strongly stable and has no generator in degree $d$. Then in $(I)$ is generated in degree $<d$.

Green shows this for the generic initial ideal gin $(I)$. However one readily sees that his argument holds under the condition that in $(I)$ is strongly stable.

2.3. Main result. Now if $T$ is a subspace of $\mathbb{k}\left[x_{1}, \ldots, x_{n}\right]$ spanned by monomials of degree $d$ and $T$ is strongly stable, then

$$
\left(T: x_{n}^{r}\right)_{\mid x_{n}} \cdot\left\langle x_{1}, \ldots, x_{n-1}\right\rangle \subseteq\left(T: x_{n}^{r-1}\right)_{\mid x_{n}} .
$$

Thus, if $I_{T}$ denotes the ideal generated by $T$, then

$$
\oplus_{r=1}^{d}\left(T: x_{n}^{r}\right)_{\mid x_{n}} \oplus\left(I_{T}\right)_{\mid x_{n}}
$$

becomes an ideal in $\mathbb{k}\left[x_{1}, \ldots, x_{n-1}\right]$ which we denote by $\mathcal{J}(T)_{x_{n}}$.

Main Theorem 2.4. Let $V$ be a subspace of $\mathbb{k}\left[x_{1}, \ldots, x_{n}\right]$ consisting of polynomials of degree $d$. Suppose $V$ is in general coordinates and let $I_{V}$ be the ideal generated by $V$.

a. If $\left(\operatorname{in}(V): x_{n}\right) \cdot\left\langle x_{1}, \ldots, x_{n}\right\rangle=\operatorname{in}(V)$ (i.e. the ideal $\mathcal{J}(\operatorname{in}(V))_{x_{n}}$ has no generator in degree $d)$, the initial ideal in $\left(\left(I_{V}\right)_{\mid x_{n}}\right)$ will be equal to this ideal in degrees $\geq d$.

b. More generally, if the ideal $\mathcal{J}\left(i n(V)_{\mid x_{n}, \ldots, x_{n-r+1}}\right)_{x_{n-r}}$ has no generator in degree $d$, the initial ideal in $\left(\left(I_{V}\right)_{\mid x_{n}, \ldots, x_{n-r}}\right)$ will be equal to this ideal in degrees $\geq d$.

Proof. a. Let $\mathbb{k}(\mathbf{t})$ be the function field of $\mathbb{k}\left[t_{1}, \ldots, t_{n}\right]$, and let $h=\sum_{i=1}^{n} t_{i} x_{i}$ in $\mathbb{k}(\mathbf{t})\left[x_{1}, \ldots, x_{n}\right]$. Also let $V_{\mathbb{k}(\mathbf{t})}=V \otimes_{\mathbb{k}} \mathbb{k}(\mathbf{t})$.

Claim. The direct sum of spaces

$$
\oplus_{r=1}^{d}\left(V_{\mathbb{k}(\mathbf{t})}: h^{r}\right)_{\mid h} \oplus\left(I_{V_{\mathbb{k}(\mathbf{t})}}\right)_{\mid h} \subseteq \mathbb{k}(\mathbf{t})\left[x_{1}, \ldots, x_{n}\right] /(h)
$$

is an ideal in the ring on the right side. 
This follows because

$$
\left\langle x_{1}, \ldots, x_{n}\right\rangle \cdot\left(V_{\mathbb{k}(\mathbf{t})}: h^{r}\right)_{\mid h} \subseteq\left(V_{\mathbb{k}(\mathbf{t})}: h^{r-1}\right)_{\mid h} .
$$

To see this let $p$ be in $\left(V_{\mathbb{k}(\mathbf{t})}: h^{r}\right)$. Then $p h^{r}$ is in $V_{\mathbb{k}(\mathbf{t})}$. Now if a vector varies in a vector space, here $V$, the derivative of the vector will also be in the vector space. Hence differentiating with respect to $\partial / \partial t_{i}$, we get

$$
\partial p / \partial t_{i} \cdot h^{r}+p \cdot r x_{i} h^{r-1} \in V_{\mathbb{k}(\mathbf{t})},
$$

and this gives that $p \cdot x_{i}$ is in $\left(V_{\mathbb{k}(\mathbf{t})}: h^{r-1}\right)_{\mid h}$. This shows the claim.

Since we assume that $V$ is in general coordinates, we can assume that $h=x_{n}$ behaves generically in the claim above. Thus

$$
I=\oplus_{r=1}^{d}\left(V: x_{n}^{r}\right)_{\mid x_{n}} \oplus\left(I_{V}\right)_{\mid x_{n}} \subseteq \mathbb{k}\left[x_{1}, \ldots, x_{n-1}\right]
$$

is an ideal. Note that $\operatorname{in}(I)$ is equal to $\mathcal{J}(\operatorname{in}(V))_{x_{n}}$.

Now we claim that in $(I)$ is strongly stable. This is so in degrees $e \geq d$ because $\operatorname{in}(I)_{e}=\operatorname{in}\left(I_{V}\right)_{\mid x_{n}, e}$. Since $V$ is in general coordinates, in $\left(I_{V}\right)$ is the generic initial ideal and so is strongly stable. But then the restriction is also strongly stable.

Now in $(I)$ is also strongly stable in degrees $\leq d$. This follows by (2) since in $(V)$ is strongly stable and so in $(V): x_{n}^{r}$, and in the end the restriction is also strongly stable.

If therefore $\mathcal{J}(\operatorname{in}(V))_{x_{n}}$ has no generators in degree $d$, we get by Green's Theorem 2.3 that $\operatorname{in}(I)$ is equal to $\mathcal{J}(\operatorname{in}(V))_{x_{n}}$, and so $\operatorname{in}\left(\left(I_{V}\right)_{\mid x_{n}}\right)$ is equal to the latter in degrees $\geq d$.

b. Let $W=V_{\mid x_{n}, \ldots, x_{n-r+1}}$. Since we are using the revlex order

$$
\operatorname{in}(W)=\operatorname{in}(V)_{\mid x_{n}, \ldots, x_{n-r+1}} .
$$

Hence we are assuming that

$$
\left(\operatorname{in}(W): x_{n-r}\right) \cdot\left\langle x_{1}, \ldots, x_{n-r}\right\rangle=\operatorname{in}(W) .
$$

But then by part a we get

$$
\mathcal{J}(\operatorname{in}(W))_{x_{n-r}}=\operatorname{in}\left(\left(I_{W}\right)_{\mid x_{n-r}}\right)
$$

in degrees $\geq d$, and this gives $\mathrm{b}$.

Corollary 2.5. a. If $\mathcal{J}(\text { in }(V))_{x_{n}}$ has codimension $c \leq n-2$, the vanishing locus of $V$ has codimension $c$ in $\mathbb{P}^{n-1}$.

b. More generally, if $\mathcal{J}\left(\operatorname{in}(V)_{\mid x_{n}, \ldots, x_{n-r+1}}\right)_{x_{n-r}}$ has codimension $c \leq n-r-2$, the vanishing locus of $V$ has codimension $c$ in $\mathbb{P}^{n-1}$.

Proof. a. Clearly $I_{V \mid x_{n}}$ and $\mathcal{J}(\operatorname{in}(V))_{x_{n}}$ have the same codimension $c$. Since $c \leq$ $n-2$, the vanishing locus of $I_{V \mid x_{n}}$ will be nonempty in $\mathbb{P}^{n-2}$. Now since $V$ is in general coordinates, $x_{n}$ may be considered a general linear form, and so $c$ is also the codimension of the vanishing locus of $V$.

b. Again $I_{V \mid x_{n}, \ldots, x_{n-r}}$ will have codimension $c$, and the vanishing locus will be nonempty in $\mathbb{P}^{n-r-2}$. Since $V$ is in general coordinates, $x_{n}, \ldots, x_{n-r}$ may be considered as general linear forms, and so $c$ is also the codimension of the vanishing locus of $V$.

Let us now show that Theorem 2.1 is also a corollary of the Main Theorem. 
Proof of Theorem 2.1. Assume that $V$ is in general coordinates and let $m=n-r$. Then

$$
\operatorname{in}(V)_{\mid x_{n}, \ldots, x_{n-r+1}}: x_{n-r}=\left\langle x_{1}, \ldots, x_{n-r}\right\rangle^{b-1} \cdot x_{1}^{a} .
$$

Hence the conditions of part $\mathrm{b}$ in the Main Theorem hold. Also since $\mathcal{J}\left(\operatorname{in}(V)_{\mid x_{n}, \ldots, x_{n-r+1}}\right)$ is the ideal generated by $x_{1}^{a}$, it will have codimension one. So if the codimension $1 \leq n-r-2=m-2$, the vanishing locus of $V$ has codimension one, and so there is a polynomial $p$ which is a common factor of the forms in $V$. Clearly $\operatorname{deg} p \leq a$. If $\operatorname{deg} p=a^{\prime}<a$ we may write $V=p \cdot V^{\prime}$ where $V^{\prime}$ is a space of forms of degree $b+a-a^{\prime}$. But then $\operatorname{in}(V)=x_{1}^{a-a^{\prime}} \cdot\left\langle x_{1}, \ldots, x_{m}\right\rangle^{b}$, and by applying the Main Theorem to $V^{\prime}$ we would again find a common factor. Continuing, taking products of common factors, we would in the end have a polynomial of degree $a$ which is a common factor.

2.4. Some examples. The Main Theorem is concerned with the situation that $\mathcal{J}(\text { in }(V))_{x_{n}}$ has no generators in degree $d$. In the following examples we discuss situations where $\mathcal{J}(\operatorname{in}(V))_{x_{n}}$ has one new generator in degree $d$ and a single generator in degrees less than $d$.

Example 2.6. Suppose $V$ is a subspace of $\mathbb{k}\left[x_{1}, x_{2}, x_{3}\right]_{3}$ and $\operatorname{gin}(V)$ is given by the following figure:

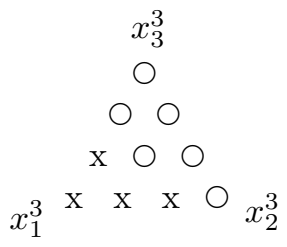

So $\operatorname{gin}(V)$ is the stable subspace generated by $x_{1}^{2} x_{3}$ and $x_{1} x_{2}^{2}$. Note that $\mathcal{J}(\operatorname{in}(V))_{x_{3}}$ has one generator of degree 2 and one generator of degree 3 . We give three examples of $V$ 's with this generic initial space.

Theorem 2.7. The following spaces $V$ will have gin $(V)$ given by the figure above.

a. $V=\left\langle x_{1} q, x_{2} q, x_{3} q, p\right\rangle$ where $q$ is a quadratic form and $p$ is a cubic form not divided by $q$. Note that if $p$ and $q$ are relatively prime, the vanishing locus of $V$ is the complete intersection of these forms.

b. $V=\left\langle x_{1}\left(x_{2}^{2}+x_{3}^{2}\right), x_{2}\left(x_{1}^{2}+x_{3}^{2}\right), x_{3}\left(x_{1}^{2}+x_{2}^{2}\right), x_{1} x_{2} x_{3}\right\rangle$. The vanishing locus of $V$ consists of the three unit coordinate vectors.

c. $V=\left\langle x_{1}^{3}+x_{2}^{3}+x_{3}^{3}, x_{1}^{2} x_{2}+x_{2}^{2} x_{3}+x_{3}^{2} x_{1}, x_{1} x_{2}^{2}+x_{2} x_{3}^{2}+x_{3} x_{1}^{2}, x_{1} x_{2} x_{3}\right\rangle$. The vanishing locus of $V$ is empty.

In particular $\mathrm{c}$ is striking in that the vanishing locus is empty even if $\mathcal{J}(\mathrm{in}(V))_{x_{3}}$ has only two generators, one in degree $d-1=2$ and one in degree $d=3$.

Proof. Note that if $\operatorname{gin}(V)$ has the form above, then $g . V: x_{3}$ is one-dimensional for a general change of coordinates $g$. Conversely, when $V$ is a four-dimensional space of forms of degree 3 with $g . V: x_{3}$ one-dimensional for a general change of coordinates, then $\operatorname{gin}(V)$ will contain $x_{1}^{2} x_{3}$. Since it is strongly stable, it must be of the form in the figure above.

Now let $\mathbb{k}(\mathbf{t})$ be the function field of $\mathbb{k}\left[t_{1}, t_{2}, t_{3}\right]$, let $V_{\mathbb{k}(\mathbf{t})}=V \otimes_{\mathbb{k}} \mathbb{k}(\mathbf{t})$ and let $h=t_{1} x_{1}+t_{2} x_{2}+t_{3} x_{3}$. We must find a four-dimensional $V$ such that $\left(V_{\mathbb{k}(\mathbf{t})}: h\right)$ 
is one-dimensional. Let this latter space be generated by a form $p$ in $\mathbb{k}(\mathbf{t})$. In $p h$, the coefficients of the monomials in $t_{1}, t_{2}, t_{3}$ must span a subspace of $\mathbb{k}\left[x_{1}, x_{2}, x_{3}\right]$ which is at most four-dimensional.

a. Suppose the t-degree of $p$ is zero. Then $p \cdot h$ is $t_{1} p x_{1}+t_{2} p x_{2}+t_{3} p x_{3}$. The coefficients here span a three-dimensional space. Adding a form $q$ of degree 3 not contained in $p \cdot\left\langle x_{1}, x_{2}, x_{3}\right\rangle$, we get a space $V=\left\langle x_{1} p, x_{2} p, x_{3} p, q\right\rangle$ whose generic initial space must be given by the figure above.

b. Suppose the t-degree of $p$ is one. Then we may write $p$ as $t_{1} p_{1}+t_{2} p_{2}+t_{3} p_{3}$ where the $p_{i}$ are polynomials in the $x$ 's. The coefficients of the monomials in the t's are

$$
x_{1} p_{1}, x_{1} p_{2}+x_{2} p_{1}, x_{1} p_{3}+x_{3} p_{1}, x_{2} p_{2}, x_{2} p_{3}+x_{3} p_{2}, x_{3} p_{3} .
$$

That these span at most a four-dimensional subspace means that there are at least two linear relations between them, or at least two linear syzygies of $p_{1}, p_{2}$, and $p_{3}$. But such $p$ 's may be found as $2 \times 2$-minors of a $2 \times 3$ matrix whose rows are these two linear syzygies. For instance we may take the matrix

$$
\left(\begin{array}{ccc}
x_{1} & x_{2} & 0 \\
0 & x_{2} & x_{3}
\end{array}\right)
$$

This gives $p_{1}=x_{2} x_{3}, p_{2}=x_{1} x_{3}$, and $p_{3}=x_{1} x_{2}$. It is then readily checked that the six polynomials in (3) span a four-dimensional vector space which is the one given in $b$.

c. Suppose the t-degree of $p$ is two. After a somewhat intricate consideration, we found that

$$
p=\left(t_{1} x_{2}+t_{2} x_{3}+t_{3} x_{1}\right)\left(t_{1} x_{3}+t_{2} x_{1}+t_{3} x_{2}\right)
$$

gives an example. It is easy to verify that the coefficients of the t-monomials in $p h$ are given by four forms generating $V$, given in c.

Note that in $\mathrm{b}$ and $\mathrm{c}$ the examples are invariant under the cyclic group $C_{3}$.

Let $p$ be a form of degree $e$ and $q$ a sufficiently general form of degree $d>e$. If $V=\left(p \cdot\left\langle x_{1}, x_{2}, x_{3}\right\rangle^{d-e}, q\right)$, then $\operatorname{gin}(V)$ is the strongly stable set of monomials generated by $x_{1}^{e} x_{3}^{d-e}$ and $x_{1}^{e-1} x_{2}^{d-e+1}$, one generator of degree $e$ and one of degree $d$. One might hope that, conversely, if $e$ is sufficiently smaller than $d$ and $\operatorname{gin}(V)$ is the above strongly stable set, this would imply that $V$ has a codimension one subspace $W$ such that $W=p \cdot\left\langle x_{1}, x_{2}, x_{3}\right\rangle^{d-e}$ for some $p$. This is equivalent to there existing a subspace $W$ such that $\operatorname{gin}(W)$ is the stable subspace generated by $x_{1}^{e} x_{3}^{d-e}$.

Example 2.8. Suppose $V$ is a subspace of $\mathbb{k}\left[x_{1}, x_{2}, x_{3}\right]_{4}$ and $\operatorname{gin}(V)$ is given by the following figure:

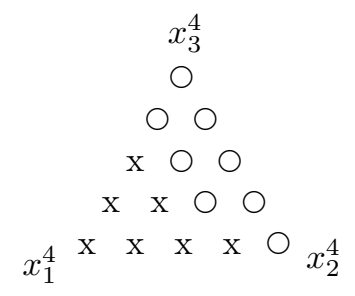


So $\operatorname{gin}(V)$ is the strongly stable subset generated by $x_{1}^{2} x_{3}^{2}$ and $x_{1} x_{2}^{3}$. Note that $\mathcal{J}(\operatorname{in}(V))_{x_{3}}$ has one generator of degree 2 and one generator of degree 4 . Could we then conclude that $V$ has a subspace $W$ of codimension one such that $\operatorname{gin}(W)$ is the stable subspace generated by $x_{1}^{2} x_{3}^{2}$ ? (This would imply that the polynomials in $W$ have a common factor.) We do not know the answer to this. But suppose $V$ is in general coordinates, so we may speak of initial ideals instead of generic initial ideals. The initial space in $\left(V: x_{3}\right)$ is

$$
\left\langle x_{1}^{3}, x_{1}^{2} x_{2}, x_{1}^{2} x_{3}\right\rangle
$$

the stable subspace generated by $x_{1}^{2} x_{3}$. If we knew that this was the generic initial space of $V: x_{3}$, we could by the techniques of Theorem 2.4 have found such a $W$. The problem is, however, that even if $V$ is in general coordinates, it is not certain that $V: x_{3}$ is, so it is not clear that $\operatorname{gin}\left(V: x_{3}\right)$ is (4). In fact, given that the dimension of $V$ is seven, the form of $\operatorname{gin}(V)$ given in the figure above is equivalent to $V: h^{2}$ being nonzero for a general $h$.

That $\operatorname{gin}\left(V: x_{3}\right)$ is given by (4) is equivalent to $\operatorname{gin}\left(V: h_{1}\right)$ being given by (4) for a general linear form $h_{1}$. But this is again equivalent to $V: h_{1} h_{2}$ being nonzero, where $h_{1}$ and $h_{2}$ are general linear forms. It may not be likely that for a sevendimensional space $V$ of forms, the condition of $V: h_{1} h_{2}$ being nonzero for general linear forms $h_{1}$ and $h_{2}$ is equivalent to the condition of $V: h^{2}$ being nonzero for a general linear form $h$.

\section{REFERENCES}

[1] D. Bayer, M. Stillman, A criterion for detecting m-regularity, Inventiones Mathematica, 87 (1987), 1-11. MR862710 (87k:13019)

[2] D. Eisenbud, Commutative algebra. With a view toward algebraic geometry. Graduate Texts in Math., 150, Springer-Verlag, 1995. MR1322960 (97a:13001)

[3] G. Fløystad, A property deducible from the generic initial ideal, Journal of Pure and Applied Algebra, 136 (1999), no. 2, 127-140. MR1674773 (2000c:13039)

[4] A. Galligo, À propos du théorème de-préparation de Weierstrass, Fonctions de Plusieurs Variables Complexes, Lecture Notes in Mathematics, 409, Springer, 1974, 543-579. MR 0402102 (53:5924)

[5] M. Green, Generic initial ideals, in Six Lectures on Commutative Algebra (Elias, J., Giral, J.M., Miró-Roig, R.M., Zarzuela, S., eds.), Progress in Mathematics, 166, Birkhäuser, 1998, 119-186. MR1648665 (99m:13040)

Matematisk Institutt, University of Bergen, Johannes Brunsgt. 12, 5008 Bergen, NORWAY

E-mail address: gunnar@mi.uib.no

Department of Mathematics, Cornell University, Ithaca, New York 14853

E-mail address: mike@math.cornell.edu 